\title{
INTERFACES IN COMPOSITE MATERIALS
}

\author{
E. Pippel and J. Woltersdorf \\ Max-Planck-Institute of Microstructure Physics, 06120 Halle, Germany
}

\begin{abstract}
The mechanical functions of the fibre/matrix interlayer as well as its quantitative parameter dependencies and their influence on the properties of the composite system are discussed, with the theoretical model being considered under realistic conditions. Corresponding to that a number of interface structure phenomena of some advanced composite materials, revealed by high voltage and high resolution electron microscopy, are discussed with respect to their relevance to the control of composite properties, including complex interlayer systems, which are produced by fibre coating as well as by chemical solid state transport and exchange reactions. The effects of hooking-together, binding, internal microcracking, and sublayer formation are demonstrated, and in some cases correlated with the mechanical behaviour of the materials as revealed by in situ tensile tests.
\end{abstract}

PACS numbers: $61.16 . \mathrm{Bg}, 68.35 . \mathrm{Fx}$; $1.60 . \mathrm{Dq}$

\section{Introduction}

As it is well known, long fibre reinforced composite materials imply a more promising damage-tolerant and quasi-ductile behaviour than monolithic ceramics even if treated in the high temperature range.

The brittleness of these composite systems under load and during crack propagation processes may be drastically reduced by activating a number of energy-dissipative mechanisms like crack shielding, crack deflection, multiple crack formation, crack branching, debonding and pull-out of the fibres. These processes are particularly operative in the interface between fibre and matrix [1, 2].

Generally, consisting of several layers with a complex microstructure, the interfacial regions are either built up by fibre coating or generated during the processing of the composites by chemical reactions.

The complex behaviour of the interfaces will be optimum with respect to debonding and pull-out processes implying the desired toughness and strength parameters, if both their microstructure and microchemistry are known and if advanced mechanical testing methods will reveal corresponding structure/property relations.

In a first part the present paper describes the mechanical functions of the interlayer between fibre and matrix; it shows their quantitative parameter dependences by the example of models currently used. Then, some results are given 
concerning the special chemistry and the process of crack propagation in such interlayer systems. Finally, valuable information on the above mentioned interface controlled toughening mechanisms will be given by scanning electron microscopic (SEM) in situ tensile tests, which combine a registration of the load/deflection behaviour during fracture with a simultaneous observation of the individual micro-processes in the composite.

Our results base on different novel composites with SiC-fibres Nicalon $($ and Tyranno $ß$ produced by polymer pyrolysis and two types of carbon fibre ( $\mathrm{T} 300$, T 800). SiC (produced by chemical vapour infiltration (CVI)) and a technical glass (boron silicate glass, Duran (B) served as matrix material.

The microstructural investigations at different structure levels down to atomic plane imaging were carried out by transmission electron microscopy (TEM), particularly high voltage (HVEM) and high resolution electron microscopy (HREM). In addition, microchemical analyses down to the nanometre range were performed by applying analytical electron microscopy (EDXS).

\section{Interface parameters and toughening process}

In fibre reinforced composite materials one of the most important parameters controlling the above mentioned energy dissipating mechanisms seems to be the shear stress $\tau$ transmitted by friction processes between fibre and matrix. The effect of this interface parameter can be demonstrated by discussing the stress/strain diagram of an unidirectional reinforced composite resulted from a tensile test as shown in Fig. 1 [3].

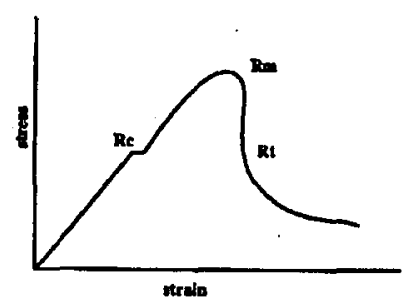

Fig. 1. Principle of the stress/strain diagram of an unidirectional fibre reinforced composite in tensile test.

Exceeding an initial linear increase in the load the first matrix cracks appear at the stationary critical matrix stress $R_{\mathrm{c}}$. As the intact fibres bridge the crack faces a further increase in the stress intensity at the crack tip is prevented and $R_{\mathrm{c}}$ can be calculated by an energy balance [4]:

$$
R_{\mathrm{c}}=\left[\frac{6 \tau \Gamma_{\mathrm{m}} V_{\mathrm{f}}^{2} E_{\mathrm{f}} E^{2}}{V_{\mathrm{m}} E_{\mathrm{m}}^{2} r_{\mathrm{f}}}\right]^{1 / 3}
$$

with $\Gamma, V, E$ and $r$ being the surface energy, volume fraction, Young moduli and radius of fibre (f) and matrix (m), respectively. 
In a similar manner the maximum stress $R_{\mathrm{m}}$ can be calculated if it is assumed that a certain shear stress is still transmitted by the embedded fibres [5]:

$$
R_{\mathrm{m}}=V_{\mathrm{f}}\left(\frac{2 \sigma m \tau l_{0}}{r_{\mathrm{f}}(m+2)}\right)^{\frac{1}{m+1}} \frac{m+1}{m+2}
$$

with $\sigma_{0}, l_{0}, m$ representing the usual Weibull parameters.

Finally, a residual strength $R_{\mathrm{t}}$ results from the sliding resistance during the fibre pull-out. If the pull-out length $\bar{h}$ is known, $R_{\mathrm{t}}$ can be calculated from

$$
R_{\mathrm{t}}=\frac{2 V_{\mathrm{f}} \tau \bar{h}}{r_{\mathrm{f}}}
$$

However, as the relations (1) to (3) base on the presupposition that there is no binding across the interface which is equivalent to finite $\tau$ values and the possibility of a well developed fibre pull-out, they will fail in case of strong interface bonding. Moreover, an extremely reduced pull-out dissipates only small amounts of energy, with the composite exhibiting a brittle fracture and a small residual strength, as deduced from (3).

Additionally, it should be mentioned that the exact value of the residual strength calculated from (3) cannot be measured in a real tensile test, as the compliance of the tensile device causes an appreciable fibre pull-out after exceeding the maximum stress. These restrictions are to be considered in the interpretation of the experimental results presented in Sec. 5 .

\section{Chemistry of interfaces}

Figure 2 shows the subdivision of the interlayer between a Nicalon fibre $(A)$ and an SiC-matrix $(E)$ : a glassy amorphous layer $(C)$ is embedded between two carbon layers of different morphology and origin $(B$ and $D)$. During debonding the layer $(C)$ is separated from the outer carbon layer $(D)$ revealing also a correspondingly porous region. High resolution investigations [6] showed that there is a continuous transition of distinct microroughness between the nanocrystalline fibre material and the strongly turbostratic, cellular $B$-layer.

Layers $B$ and $C$ obviously arise from a fibre degradation caused by oxygen diffusion from the matrix material (see below).

The outer boundary of the layer system, i.e. the $D / E$ interface, is determined by initial processes of the CVI-SiC growth, which mainly proceeds by dendrites normal to the interface $(E)$.

Structure and composition of this interlayer as well as the properties of the composite drastically change after high-temperature treatment $\left(1200^{\circ} \mathrm{C}\right)$ in air: interlayer oxidation, mainly caused by pipe diffusion of oxygen, initiates the formation of glassy interface regions ( 1 and 2 in Fig. 3), which are separated by a carbon layer of pores and voids, and which cause a stronger bonding to fibre and matrix, respectively. In accordance with Sec. 2 mechanical tests showed a correspondingly insufficient pull-out behaviour and failure by brittle fracture.

The interlayer between Nicalon fibre and Duran glass matrix seems to be of particular interest: as shown in Fig. 4, it consists of a carbon layer (2) of about $60 \mathrm{~nm}$ in width and has a cellular graphitic morphology. On its fibre side there is 


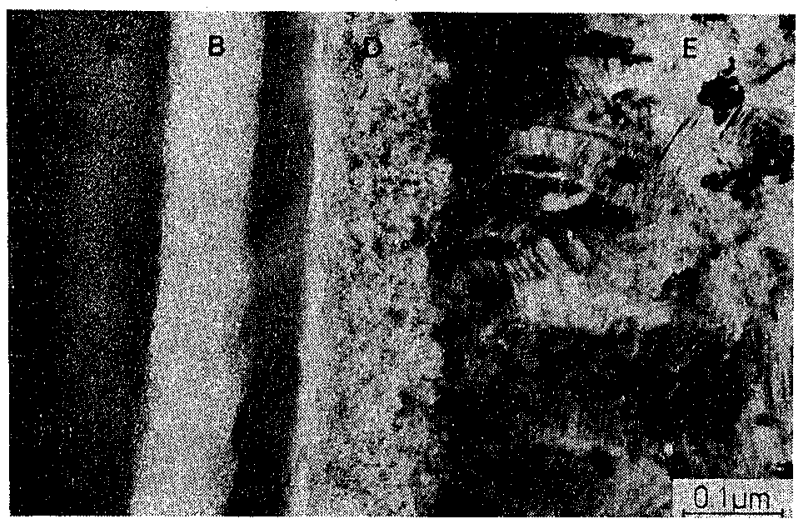

Fig. 2. Layered structure of the interface: $A$ - Nicalon fibre, $B$ - inner carbon layer, $C-\mathrm{SiO}_{2}$ layer, $D-$ outer carbon layer, $E-\mathrm{SiC}$-matrix.

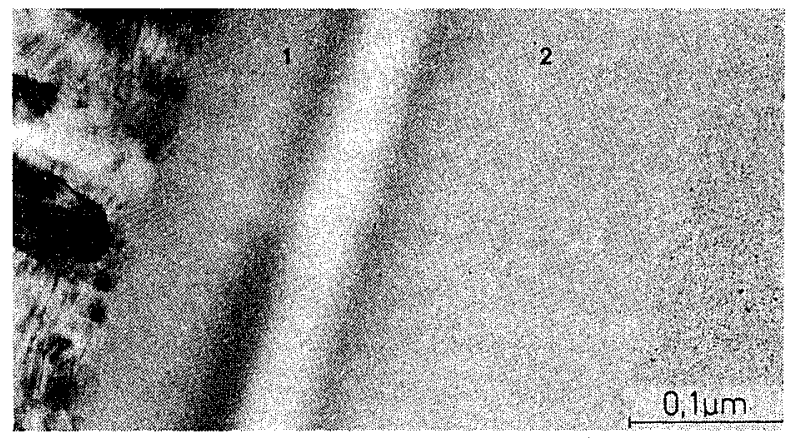

Fig. 3. Glassy layers $(1,2)$ in the fibre/matrix interface of $\mathrm{SiC} / \mathrm{SiC}$ after high-temperature treatment $\left(1200^{\circ} \mathrm{C}\right)$ in air.

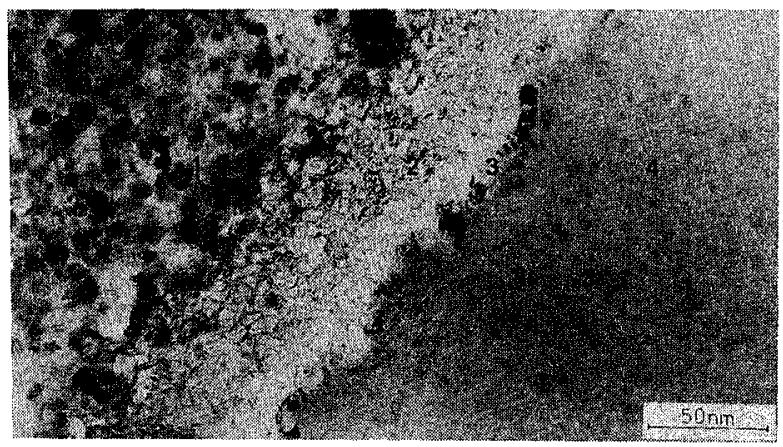

Fig. 4. Interlayer structure of a Nicalon/Duran composite: 1 - fibre, 2 - cellular region of the C-layer, 3 - ribbon of graphite, 4 - matrix. 
an irregular boundary, whereas on the matrix side there is a ribbon of regularly arranged atomic planes of graphite (3) in distinct interface-parallel orientation. These structural peculiarities are due to interface reactions. Pressing time and temperature strongly determine the degree of their appearance. Because of their influence on the fibre pull-out as well as on the residual stresses in the composite microchemical transport reactions in the interface region were examined in more detail.

The high resolution EDXS-analysis (IIR STEM HB 501, Kevex Delta III) of the microchemistry of interlayers, as shown in Fig. 4, yielded the following: $\mathrm{Si}$ and $\mathrm{O}$-signals abruptly decrease at the very border of the matrix/C-layer (cf. Fig. 5) of a shortiy tempered sample $\left(5 \mathrm{~min}\right.$ at $1240^{\circ} \mathrm{C}$ ). The layer itself (box-like profile) clearly consists of almost solely carbon with only traces of $\mathrm{Si}$ and $\mathrm{O}$. If the carbon occurrence results from the oxidation reaction [7] of $\mathrm{SiC}+\mathrm{O}_{2}=\mathrm{SiO}_{2}+\mathrm{C}$, then $\mathrm{SiO}_{2}$ simultaneously generated in this case should have diffused in the direction of the matrix and dissolved there. In the region of its matrix-near graphite layer

Fig.5
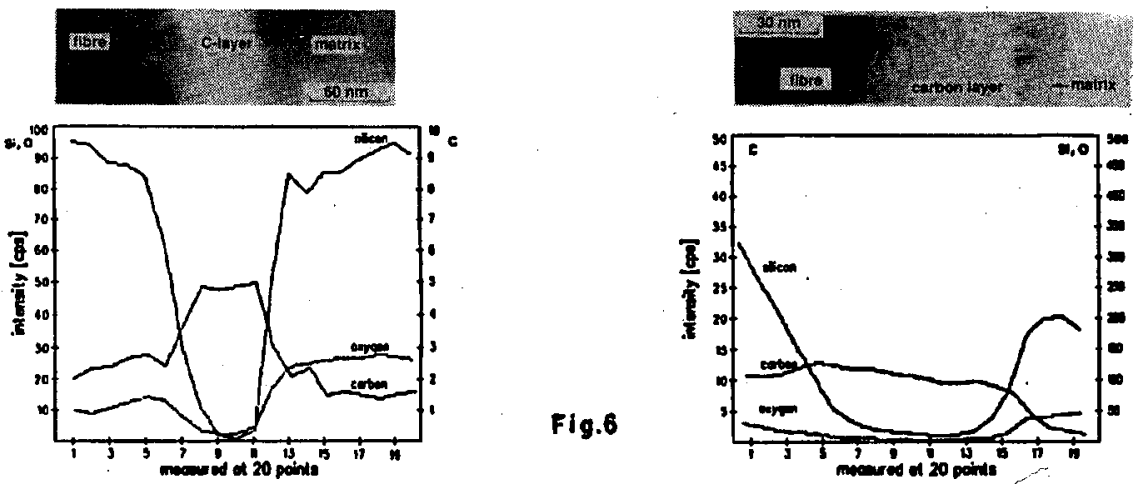

Fig.6

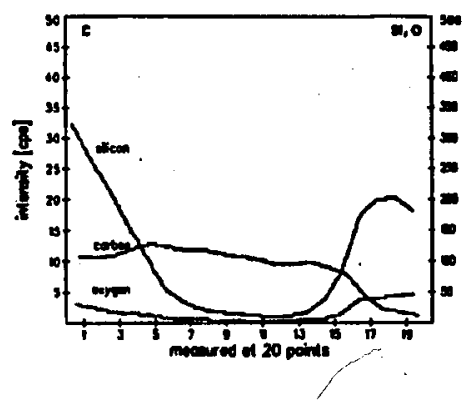

Fig. 5. EDXS-analysis of the reaction interlayer in a Nicalon/Duran composite, hot-pressed for $5 \mathrm{~min}$ at $1240^{\circ} \mathrm{C}$

Fig. 6. EDXS-analysis of the reaction interlayer in a Nicalon/Duran composite, hot-pressed for $4 \mathrm{~h}$ at $1300^{\circ} \mathrm{C}$.

a long-tempered sample $\left(4 \mathrm{~h}\right.$ at $\left.1300^{\circ} \mathrm{C}\right)$ unambiguously shows fractions of $\mathrm{Si}$ and $O$ (see Fig. 6), which the first sample displays only within the matrix. This may indicate that the oriented graphite layer ((3) in Fig. 4) serves as a barrier to the diffusion of $\mathrm{SiO}_{x}$ from the C-layer into the glass matrix. Owing to the strongly anisotropic graphite bond normal to the hexagonal basal planes of graphite, diffusion of impurities as well as self-diffusion are slower than within the planes [8]. Therefore, the reaction products may diffuse only between the basal planes, i.e. along the graphitic structure and its lamellae (within the cellular part of the interlayer), or along the ribbons of regular atomic planes (at the layer/matrix interface). 


\section{Crack processes at interfaces}

Besides the thermomechanical and chemical accommodation of interfaces in advanced composite materials, their most important functions are to protect against oxidation in the high-temperature range and to enable an appropriate mechanical gliding behaviour. Here, we will only deal with the latter one: if the bonding across the interface is too strong, a matrix crack may cause a brittle failure of the material. Very weak interactions, however, prevent the load transfer from the fibre to the matrix, with only a small part of energy being dissipated during the pull-out process.

The following two micrographs point out the influence of ribbons of graphite atomic planes parallel to the interface on debonding and pull-out processes.

Here, the coating of a Tyranno fibre with pyrolytic carbon in an SiC-matrix caused a cellular turbostratic layer of graphite ribbons to form (cf. Fig. 7). Along the very boundary to the fibre a $10-20 \mathrm{~nm}$ thick ribbon of graphite atomic planes is

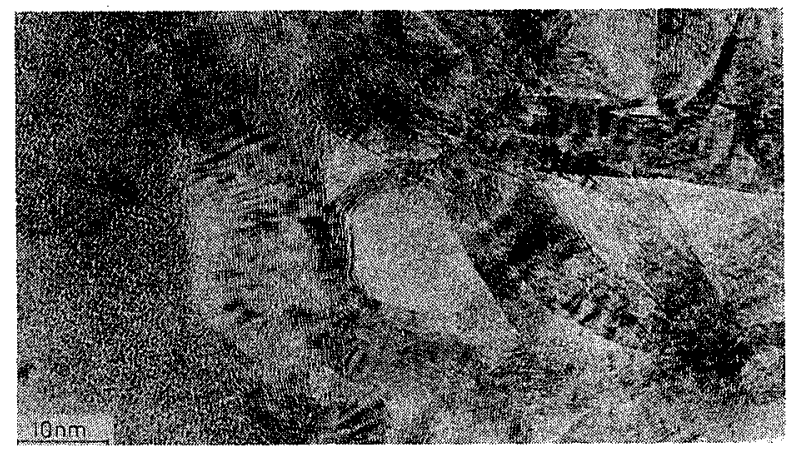

Fig. 7. Carbon layer of a Tyranno fibre: alignment of the graphite structure on approaching the fibre surface (left).

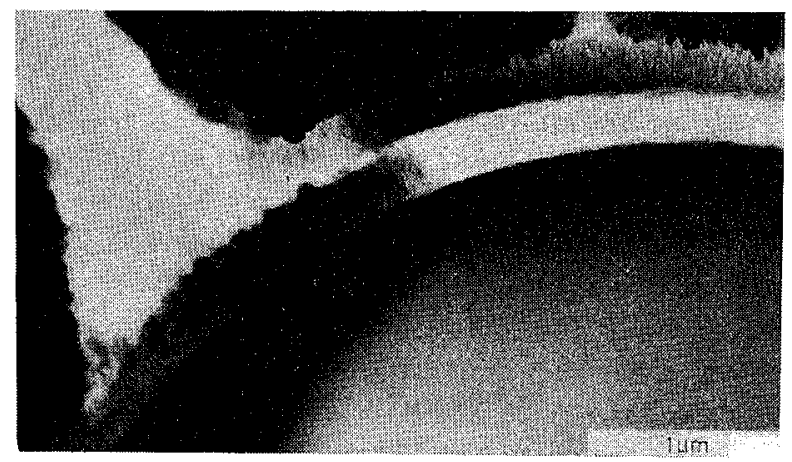

Fig. 8. Crack propagation along the interface between fibre (below) and graphite layer in $\mathrm{SiC} / \mathrm{SiC}$. 
aligned parallel to the fibre surface (left). Figure 8 reveals the effect: the microcrack starting in the upper left takes off the inner side of the interlayer from the fibre, i.e. in the interlayer the crack propagates within the very region of graphite atomic planes aligned parallel to the interface, shown in Fig. 7.

The interface-parallel ribbon of graphite atomic planes hence acts as a solid lubricant. Obvious reasons for that are particular bonding conditions and anisotropies of graphite: atoms of hexagonal basal planes of graphite are linked up by strong localized bonds of about $140 \mathrm{kcal} / \mathrm{mol}^{-1}$ which are built of $2 s, 2 p_{x}$ and $2 p_{y}$ electrons. Contrary, the bond between two basal planes originates rather from the non-vanishing overlap integral of the $\pi$-electrons. It may be denominated intermolecular cohesion and is very weak, enabling ideal gliding between the atomic planes. Hence, the parallel enveloping of the fibre in such atomic planes should allow a quasi-laminar pull-out procedure and explain the cracks, which we often observed along the layer/fibre interface. Furthermore, this observation suggests that the fibre resistance to glide, which is an important parameter of the interface frictional stress dealt with in Sec. 2, is controlled by the variable formation and decomposition of laminar interface regions, between fibre and entangled structure of the layer.

The described interface mechanism is not restricted to either the special fibre or the matrix material; it only demands the development of a properly oriented graphite layer. As a further example, Fig. 9 shows the typical interlayer structure of a T 300 carbon fibre reinforced CVI-grown SiC ceramic. On the very fibre surface (above) a parallel-oriented graphitic band has formed (dark contrast). Furthermore, the excellent hooking-together of the cellular-structured carbon layer with the first matrix crystallites can be observed (below). Owing to the special binding conditions in graphite, i.e., the relatively small interactions between the hexagonal basal planes (cf. above), such a configuration should enable a quasi-laminar pull-out. This is demonstrated by the debonding crack propagating along the graphitic band, shown in the figure (middle part).

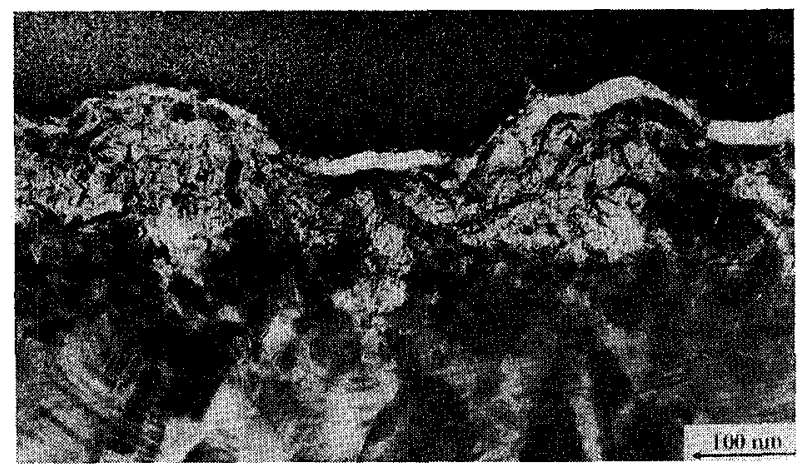

Fig. 9. Interlayer structure between a carbon fibre (above) and the SiC-matrix including a debonding crack (middle part). 


\section{In situ tensile experiments}

To establish a correlation between fracture behaviour and microstructure, as well as to study the influence of the processing temperature on the interface structure, in situ tensile tests were carried out using a special micro tensile table, which was incorporated in a ESEM-3 scanning electron microscope. Two unidirectional reinforced Nicalon/Duran composites, hot-pressed for 5 minutes at about $1270^{\circ} \mathrm{C}$ (specimen $A$ ) and $1100^{\circ} \mathrm{C}$ (specimen $B$ ), respectively, were investigated.

A characteristic load/displacement diagram of specimen $A$ processed at the higher temperature is shown in Fig. 10. Starting with a nearly linear-elastic range, the slope of the curve decreases well before the maximum load. In this region increasing matrix fracture, debonding processes, as well as a rupture of the first fibres were observed. After exceeding the maximum, the load abruptly declines; this effect is connected with the rupture of nearly all fibres. The appreciable residual load of more than $50 \%$ of the maximum is carried now by the frictional shear between fibres and matrix. The further straining of the composite is characterised by an extensive stick-slip behaviour, i.e., a successive pull-out with an amplitude proportional to the residual load. This implies the stick-slip is mainly caused by the compliance of the tensile stage. From the measured high residual load and the large amount of energy dissipating pulled out fibres (cf. inset of Fig. 10), a high toughness of this material can be concluded. The ability of extensive fibre pull-out in specimen $A$ is also revealed by HREM observations of the interface region: as shown on the lattice fringe image in Fig. 11a, a cellular configurated carbon-rich layer has been formed between fibre and matrix by oxidation of the fibre $\mathrm{SiC}$, which should determine the details of the composite micro mechanisms under load and during failure [9]. Moreover, a well ordered ribbon of parallel oriented atomic

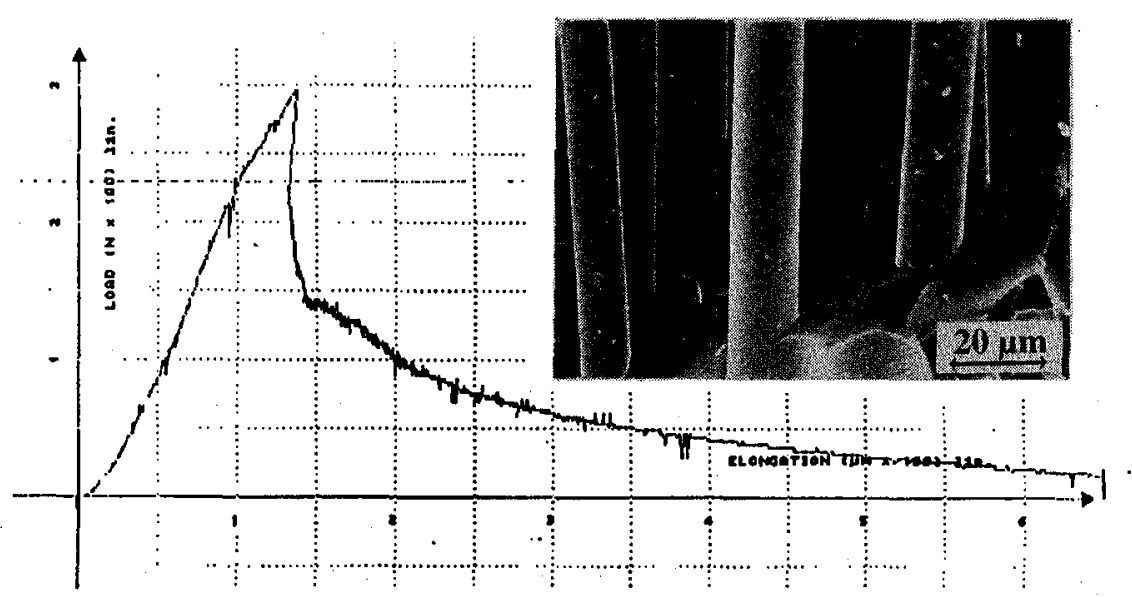

Fig. 10. Load/displacement diagram of a Nicalon/Duran composite pressed at $1270^{\circ} \mathrm{C}$ (inset: SEM image of the fracture surface). 


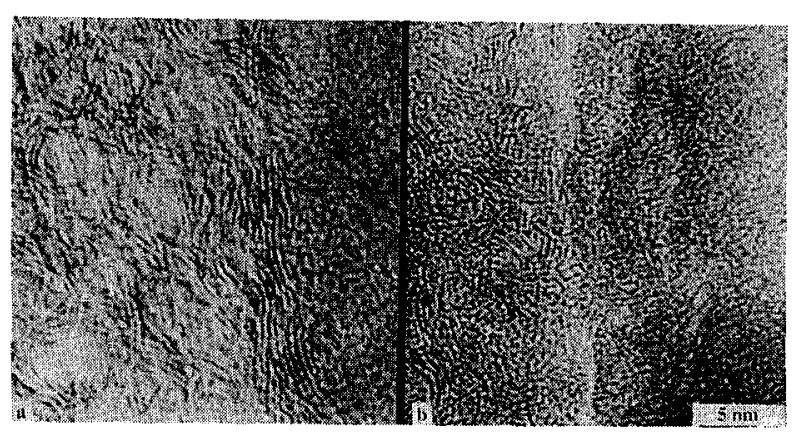

Fig. 11. HREM images of the carbon interlayer structures in Nicalon/Duran composites hot-pressed at (a) $1270^{\circ} \mathrm{C}$ and (b) $1100^{\circ} \mathrm{C}$.

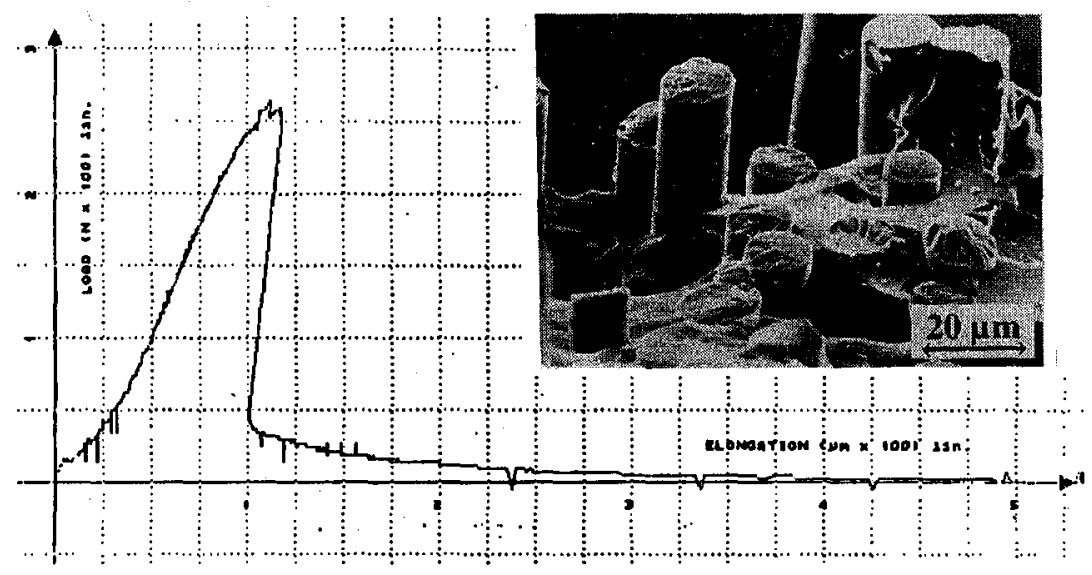

Fig. 12. Load/displacement diagram of a Nicalon/Duran composite pressed at $1100^{\circ} \mathrm{C}$ (inset: SEM image of the fracture surface).

planes of graphite was found at the interface near to the matrix (cf. Fig. 11a right part). According to the statement in Sec. 4 these carbon planes can effect a quasi-laminar pull-out, thus acting as a solid lubricant.

Contrary to this, specimen $B$ processed at a lower temperature exhibits a somewhat different structure of the carbon-rich interlayer. In particular, as shown in Fig. 11b, the cellular structure is less developed and the aligned graphite band is completely absent. The missing graphitic lubricant layer should increase the frictional and even more the bonding forces in the interface. Consequently, a suppressed fibre pull-out, i.e., a more brittle composite behaviour should result. Exactly this was observed in the tensile tests of specimen $B$ : as can be seen on the adequate load/displacement diagram in Fig. 12, after exceeding the maximum a large load drop of more than $70 \%$ was found. Because of the strongly bonded fibres 
in this material the matrix cracks are hindered to be deflected in the interfaces and only small pull-out lengths can be realized (cf. the inset in Fig. 12). Although the frictional shear stress $\tau$ of the interface may be slightly increased with respect to specimen $A$, the drastically decreased a verage pull-out length of material $B$ leads to the observed low residual strength as described by Eq. (3) in Sec. 2.

\section{Acknowledgments}

Thanks are due to the Deutsche Forschungsgemeinschaft for financial support, to Schott, Mainz, for providing the materials, to the Fraunhofer Institute for Mechanics of Materials, Branch lab Halle, for the facilities of ESEM 3 / tensile stage and to R. Schneider for help in microchemical analyses.

\section{References}

[1] A.G. Evans, D.B. Marshall, Acta Metall. 37, 2567 (1989).

[2] E. Bischoff, M. Rühle, O. Sbaizero, A.G. Evans, J. Am. Ceram. Soc. 72, 741 (1989).

[3] G. Grathwohl, M. Kuntz, E. Pippel, J. Woltersdorf, Phys. Status Solidi 146, 393 (1994).

[4] L.N. McCartney, Proc. R. Soc. London 408, 329 (1987).

[5] W.A. Curtin, J. Am. Ceram. Soc. 74, 2837 (1991).

[6] J. Woltersdorf, E. Pippel, Proc. 2nd. Symp. Mater. Sci. of BMFT, Vol. 2, Verl. TÜV Rheinland, Köln 1991, p. 1768.

[7] R.F. Cooper, K. Chyung, J. Mater. Sci. 22, 3148 (1987).

[8] B.T. Kelly, Physics of Graphite, Appl. Sci. Publ., London 1981.

[9] M. Monthioux, Revue des composites et de materiaux avances 3, 70 (1993). 